\title{
A quantitative analysis of transmission efficiency versus intensity for malaria
}

\author{
David L. Smith,2, Chris J. Drakeley³, Christinah Chiyaka' \& Simon I. Hay ${ }^{4}$
}

The relationship between malaria transmission intensity and efficiency is important for malaria epidemiology, for the design of randomized control trials that measure transmission or incidence as end points, and for measuring and modelling malaria transmission and control. Five kinds of studies published over the past century were assembled and reanalysed to quantify malaria transmission efficiency and describe its relation to transmission intensity, to understand the causes of inefficient transmission and to identify functions suitable for modelling mosquitoborne disease transmission. In this study, we show that these studies trace a strongly nonlinear relationship between malaria transmission intensity and efficiency that is parsimoniously described by a model of heterogeneous biting. When many infectious bites are concentrated on a few people, infections and parasite population structure will be highly aggregated affecting the immunoepidemiology of malaria, the evolutionary ecology of parasite life history traits and the measurement and stratification of transmission for control using entomological and epidemiological data.

\footnotetext{
${ }^{1}$ Emerging Pathogens Institute, University of Florida, Gainesville, Florida 32610, USA. ${ }^{2}$ Department of Biology, University of Florida, Gainesville, Florida 32611, USA. ${ }^{3}$ Department of Infectious \& Tropical Diseases, London School of Hygiene and Tropical Medicine, London WC1E 7HT, UK. ${ }^{4}$ Spatial Ecology and Epidemiology Group, Department of Zoology, University of Oxford, Tinbergen Building, South Parks Road, Oxford OX1 3PS, UK. Correspondence and requests for materials should be addressed to D.L.S. (email: smitdave@gmail.com).
} 
M alaria, dengue, filariasis and other mosquito-borne diseases cause several hundred million cases of clinical disease and more than one million deaths each year. A theory of mosquito-borne disease transmission and control has been developed around a mathematical model described by Ross, analysed by Lotka, and parameterized and applied by Macdonald ${ }^{1-3}$. The theory describes the potential intensity of transmission and the dynamics of mosquito-borne diseases in terms of the basic reproductive number $\mathrm{R}_{0}$, the number of infectious mosquitoes that would arise from a single infectious mosquito after one parasite generation in an immunologically naive population ${ }^{2,4}$. A formula for $\mathrm{R}_{0}$ is based largely on entomological aspects of transmission described by vectorial capacity, the number of infectious bites that would eventually arise from all the mosquitoes that bite a fully infectious human on 1 day $^{5} . \mathrm{R}_{0}$ describes a threshold for endemic persistence of malaria and other weakly immunizing mosquito-borne infections, a threshold for epidemic spread of strongly immunizing pathogens, the rate of increase in the number of cases during an epidemic ${ }^{6}$ and a basis for setting transmission reduction targets that must be achieved through vector control to eliminate endemic transmission or prevent epidemics ${ }^{4,7,8} \cdot \mathrm{R}_{0}$ can be estimated directly through entomological studies and vectorial capacity, or it can be estimated indirectly using epidemiological, serological or entomological measures of transmission ${ }^{4,9}$.

Two serious challenges to the quantitative validity of using entomological metrics for control were raised by studies that obtained very different indirect estimates of $\mathrm{R}_{0}$ using different measures of transmission ${ }^{10,11}$. One measure of transmission is the average number of infectious bites per person per unit time, called the entomological inoculation rate (EIR). Another measure of transmission is the number of infections per person per unit time, called the force of infection (FOI). The FOI counts all incident (that is, new) human malaria infections in some time interval with or without clinical symptoms, and whether or not a person is already infected. The number of infections per infectious bite (FOI/EIR) describes the efficiency of transmission. A third measure of transmission is the sero-conversion rate (SCR), the rate that humans develop serological responses to the products of parasite infections; the SCR is analogous to the FOI and would be very similar if serological responses were highly predictive of previous infection. Several studies that have estimated transmission efficiency have shown that malaria transmission is extremely inefficient in high-intensity settings ${ }^{10,12-18}$, and there has been a long-standing debate about why ${ }^{17-22}$. Low transmission efficiency gave rise to the challenges about the quantitative validity of using entomological measures as a basis for measuring transmission and planning control.

One reason for inefficient malaria transmission is that the parasites may not be transmitted from an infectious mosquito to a human during the blood meal. Once in a human or other vertebrate host, transmitted sporozoites could also fail to advance to the blood stages by chance. Some infections may be prevented by an acquired pre-erythrocytic immunity to malaria, but despite evidence that immunity to pre-erythrocytic stages can be induced artificially, there is very little direct evidence that pre-erythrocytic immunity blocks a high fraction of incoming infections ${ }^{23}$. Immunity to blood stages of the parasites, especially in those who have well-developed bloodstage immunity, could also cause inefficient transmission by rapidly clearing primary merozoites or suppressing parasite densities below levels that are detectable by microscopy, thereby preventing patent (that is, detectable) infections ${ }^{19,24}$. It has also been proposed that an acute immune response to mosquito bites could confer short-term protection against infection ${ }^{25}$. A variety of other explanations have been proposed to explain inefficient transmission in infants, such as fetal haemoglobin, changes in the intestinal flora, the predominance of a milk-based diet and maternal antibodies, but these would not explain inefficient transmission observed in older children ${ }^{20}$.
Another explanation is heterogeneous biting, in which a few people receive most of the infectious bites ${ }^{18,26,27}$. Contrast homogenous biting, in which 10 different people each get 1 infectious bite, with heterogeneous biting, in which 1 infectious person gets 10 infectious bites and the other 9 get none. Infections are not directly observable, so by most methods, heterogeneous biting would make it appear that there are many fewer infections than infectious bites. Transmission would also appear to be inefficient if the estimates of the EIR were biased, such that estimates of the EIR were always higher than the actual EIR. The critical issues are the degree of transmission efficiency, its quantitative relationship to the EIR, and the underlying cause of the apparent transmission inefficiency, whether it is heterogeneous biting, immunity or measurement bias. These causes are not mutually exclusive; each factor could explain some part of the discrepancy.

What remains unclear is what the apparent inefficiency of malaria transmission means for the control of malaria and other mosquitoborne diseases. Two key assumptions of the basic theory of mosquito-borne transmission are that the potential intensity of transmission is related to the vectorial capacity ${ }^{5}$, and that the number of human infections that occurs is proportional to the number of infectious bites. The studies of malaria provide a chance to test the mosquito-based theory to evaluate whether the cause of inefficient transmission is likely to be unique to malaria, or whether these same patterns would likely apply in other settings and to other mosquitoborne diseases.

In this study, we have assembled and analysed malaria data to look for broad patterns, such as systematic differences in the efficiency of transmission across the spectrum of transmission intensity. The assembled data trace a strongly nonlinear relationship between transmission efficiency and transmission intensity; the number of infectious bites ranged up to several hundred per year, but the number of infections rapidly saturates when the annual EIR is $>10$. The patterns suggest that immunity does not explain inefficient transmission in young children, but that a model of heterogeneous biting does. These results will affect the way commonly measured malaria metrics are used to stratify transmission to plan for and evaluate the outcome of malaria control programmes.

\section{Results}

Literature search. A preliminary literature search identified five kinds of studies that estimated transmission efficiency either directly by exposing people to the bites of infectious mosquitoes, or indirectly as the ratio of the FOI and the EIR, the number of infections per infectious bite: (1) human subjects were challenged by exposing them to the bites of infectious mosquitoes ${ }^{28-31} ;(2)$ synthetic cohorts of uninfected people were created by curing infections with antimalarial drugs, and the cohorts were followed over time to estimate the attack rate: the proportion of the cohort that was infected ${ }^{16,32,33}$; (3) cross-sectional parasite surveys were used to estimate the FOI by fitting models to the rise in malaria prevalence with age, after accounting for infections that were cleared ${ }^{12-14}$; (4) cross-sectional serological surveys were used to estimate the SCR by fitting models to the rise in seroprevalence with age, after accounting for waning immunity (or sero-reconversion) ) $^{34,35}$; and (5) longitudinal studies were used to estimate the FOI by following individuals over time as they naturally acquired infections, and several study designs and methods were used to infer the attack rates from a sequence of parasite positive or negative observations ${ }^{15,36,37}$. These studies were reanalysed and the results assembled to evaluate the functional relationship between the FOI (denoted $h$ in equations, which Ross called the 'happenings' rate $^{1}$ ) and the EIR (denoted $E$ in equations). The efficiency of transmission (that is, $h / E$ ) was also plotted as a function of the EIR.

Experimental challenge. One source of the discrepancy between the EIR and the FOI is the proportion of infectious bites that cause 
a patent infection in a population previously unexposed to malaria, denoted as $b$. A nearly ideal natural experiment to estimate $b$ has been conducted ethically by experimental challenge to infectious mosquitoes in the non-intervention arms of drug or vaccine trials on subjects who had no previous malaria exposure $e^{29-31}$. Data from these trials were assembled and analysed (Table 1; Supplementary Note 1).

The analysis assumed independent infection probabilities: the probability of infection after being challenged with $n$ infectious bites was $1-(1-b)^{n}$. Some trials report a range for the number of infectious mosquitoes in the challenge, so maximum likelihood analysis to estimate $b$ was performed in four ways (Fig. 1). Maximum likelihood estimation (MLE) estimates of $b$ were as follows: $54 \%$ (47-62\%) using only those data that reported the exact number of challenge mosquitoes; $55 \%(64-48 \%)$ using the lower bound on the number of bites; $54 \%$ (47-63\%) using the upper bound on the number of bites; and 55\% (47-63\%) by averaging the log likelihood of the lower and upper bounds.

A synthetic cohort study. Analysis of synthetic cohorts was carried out in Saradidi, Kenya, in 1986-1987 to provide baseline epidemiological information prior for a vaccine control trial ${ }^{16}$. Every month, a new cohort of $\sim 40$ children was drawn at random from the

Table 1 | The outcome of experimental challenge to the bites of infectious mosquitoes.

\begin{tabular}{|lcccccc|}
$\begin{array}{l}\text { Number of } \\
\text { infectious bites }\end{array}$ & $\mathbf{1}$ & $\mathbf{2}$ & $\mathbf{5}$ & $\mathbf{4 - 7}$ & $\mathbf{5 - 8}$ & $\mathbf{6 - 1 1}$ \\
\hline $\begin{array}{l}\text { Number infected } \\
\text { Number challenged }\end{array}$ & 5 & 6 & 215 & 20 & 1 & 3 \\
\hline
\end{tabular}

The number of infectious bites varied over the course of more than 20 years of experiments; it is now commonly performed with five infectious bites. The columns report the number of bites, the total number of people who were infected and the total number of people who were challenged. A detailed description of the data is available in the Supplementary Information.

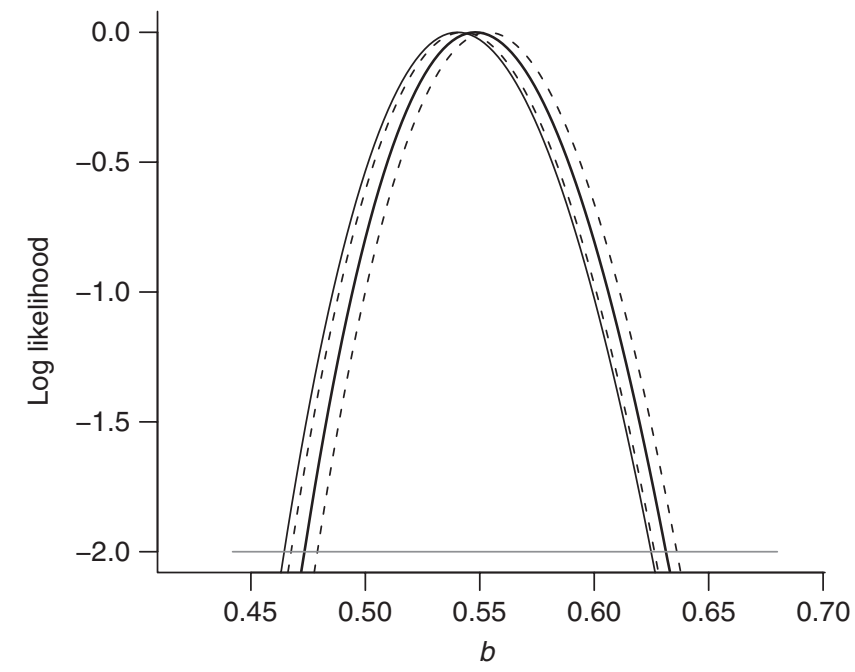

Figure 1 | The log likelihood of the proportion infected per bite after experimental challenge to infectious mosquitoes. Data were assembled from the non-intervention arms of vaccine and drug trials in carefully monitored studies using human subjects previously unexposed to malaria (Table 1). The thin solid line uses only the data for which an exact number of infectious bites were reported. The two dashed lines use the upper or lower bound or the upper bound for the number of infectious bites. The solid line uses the average of the log likelihood from the upper and lower bound. In people previously unexposed to malaria, the ratio of the $\mathrm{FOI}$ to the EIR is $\sim 55 \%$, with confidence limits between 47 and $63 \%$. whole population, and their malaria infections were cleared with the antimalarial drug sulphadoxine-pyrimethamine. After waiting a month until the drugs had cleared, the cohort was followed for 2 more months. The EIR was measured by human landing catches in each 2-week period and it was then compared with the attack rate: the proportion of the population that became infected in the subsequent 2 -week period. Variability in the EIR over 44 consecutive 2 -week periods was reflected in variable incidence in the 21 different cohorts, and this provided a way of estimating transmission efficiency over time, while the daily EIR was measured at values ranging from zero up to five infectious bites per person per day.

Three models were fit to these data. First, the FOI in a population with a given EIR and a fixed level of immunity would be given by the equation $h=b E$; this is the assumption made by the Ross-Macdonald model. Second, in the original study from Saradidi, the data were described by a linear relationship: $h=h_{0}+b E$. This unrooted linear model thus predicts that transmission still occurs at the rate $h_{0}$ even when the EIR is actually zero. Third, heterogeneous biting could explain the observed relationship between the EIR and the $\mathrm{FOI}^{18}$. With Gamma-distributed biting rates (Methods), a nonlinear relationship between the EIR and the FOI is given by the equation:

$$
h=\frac{\log (1+\alpha b E t)}{\alpha t}
$$

When biting is more heterogeneous, the estimated FOI is lower relative to the EIR.

The linear models and Equation 1 were fitted to the data from Saradidi using MLE and ranked by Akaike's information criterion (AIC, Table 2, Fig. 2). The rooted linear relationship fits very poorly. The unrooted linear function provides the best fit to the data; the fitted values could be interpreted to mean that the actual EIR was less variable and more like the overall average in the synthetic cohort compared with the estimated EIR.

The heterogeneous biting model ranked second, but with a low $\triangle \mathrm{AIC}$ value. The MLE of $\alpha \approx 4.6$ (confidence interval (CI): 3.9-5.4) corresponds roughly to the proposed rule that $20 \%$ of the population receives $80 \%$ of the bites ${ }^{26,27}$. The MLE of $b$ was $27 \%$ (CI: $21-$ $35 \%$, Fig. 2b), approximately one-half of the value found using the vaccine-trial data. In Saradidi, when only one infectious bite was counted during a 2-week period (the lowest positive count), 63/392 people (16.1\%, CI: $12.6-20.1 \%)$ were infected in the subsequent 2week period. Notably, when the EIR was estimated to be zero in the previous 2-week period, 63/349 people were infected for an attack rate of $18.1 \%$ (CI: $14.2-22.5 \%)$.

A sensitivity analysis considered the potential effect of EIR measurement bias on the parameter estimates (Methods). A bias of 2 is in line with the analysis from the experimental challenges (Fig. 2c). Models were also developed to test the idea that short-term immunity was affecting the outcome by looking for an effect of the EIR in the previous $2-4,4-6,6-8$ and $8-10$ weeks. The model fits suggested

Table 2 | Results of the reanalysis of the synthetic cohort study.

\begin{tabular}{|c|c|c|c|c|}
\hline \multirow{2}{*}{$\begin{array}{l}\text { Function } \\
h / E\end{array}$} & \multicolumn{3}{|c|}{ Fitted parameters } & \multirow[t]{2}{*}{$\Delta \mathbf{A I C}$} \\
\hline & $\boldsymbol{b}$ & Other & $\theta$ & \\
\hline$b$ & $1 / 20$ & - & 8.1 & 47 \\
\hline$\frac{h_{0}}{E}+b$ & $1 / 37$ & $h_{0} \approx 0.015$ & 11.8 & 0 \\
\hline$\frac{\log (1+b E \alpha t)}{E \alpha t}$ & 0.27 & $\alpha \approx 4.6$ & 11.8 & 1.5 \\
\hline \multicolumn{5}{|c|}{$\begin{array}{l}\text { The first column shows the function describing a relationship between EIR and transmission } \\
\text { efficiency, } h / E \text {, that was fitted to the data. The next three columns show the fitted parameter } \\
\text { estimates. The parameter } b \text { means the same in all the models, and the parameter } \theta \text { accounts for } \\
\text { extra-binomial variation. The final column shows the } \Delta A I C \text { values, that is, the AIC values relative } \\
\text { to the minimum. }\end{array}$} \\
\hline
\end{tabular}




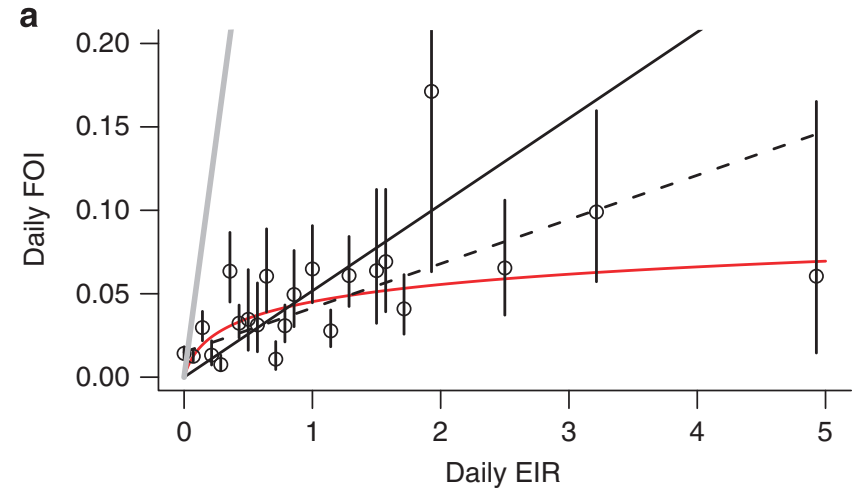

b

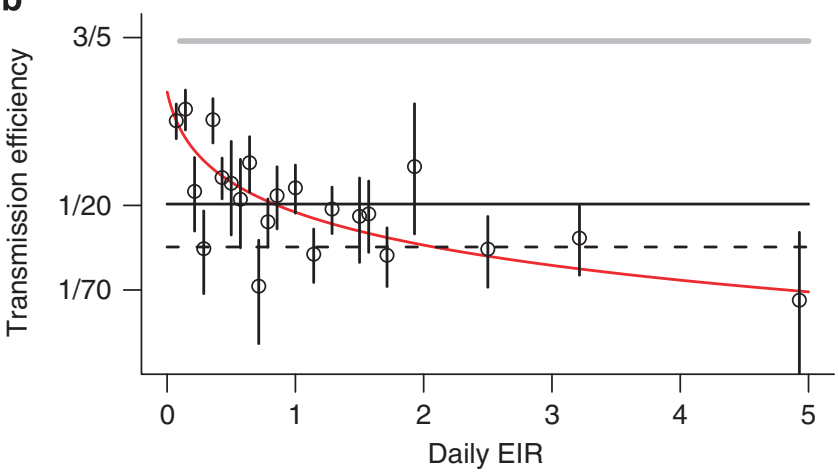

C

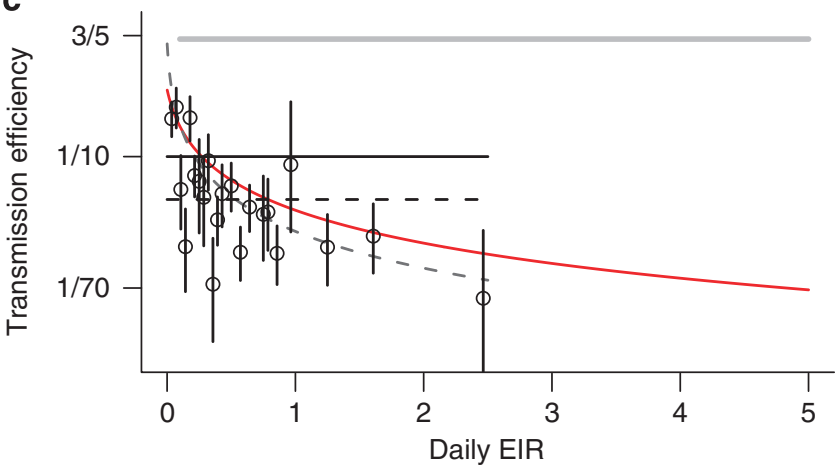

Figure $\mathbf{2}$ | Data and fitted models from the reanalysis of the synthetic cohort study. The top two panels have replotted the original data and results of the reanalysis in two ways: (a) the daily FOI and (b) transmission efficiency versus the daily EIR. Each point is an estimate of the daily FOI or transmission efficiency; the bars show the confidence intervals on the FOI by the exact test on the attack rate. Lines show the best fits for the fitted models: solid black is the rooted linear model $(b \approx 1 / 20)$, dashed black shows the slope from the unrooted linear model $(b \approx 1 / 37)$ and red shows the best-fit heterogeneous biting model. The grey line is plotted for reference to show the relationship predicted by the Ross-Macdonald model (that is, a linear relationship), assuming $b=0.55$. (c) Under the assumption that EIR was biased by a factor of two, the analysis was redone and plotted as transmission efficiency. The fitted slopes for the rooted (solid black) and unrooted (dashed black) linear models are different $(b \approx 1 / 10$ or $1 / 20$, respectively). The dashed red line shows the new best fit for the heterogeneous biting model. The solid red line and grey lines were replotted from $\mathbf{b}$ for reference.

a slightly protective effect associated with biting 6-8 weeks previously, but not with biting 2-6 weeks or 8-10 weeks previously.

Cross-sectional parasite surveys. Some of the most readily available data for estimating the FOI comes from cross-sectional parasite surveys that report the prevalence of infection, also called the parasite
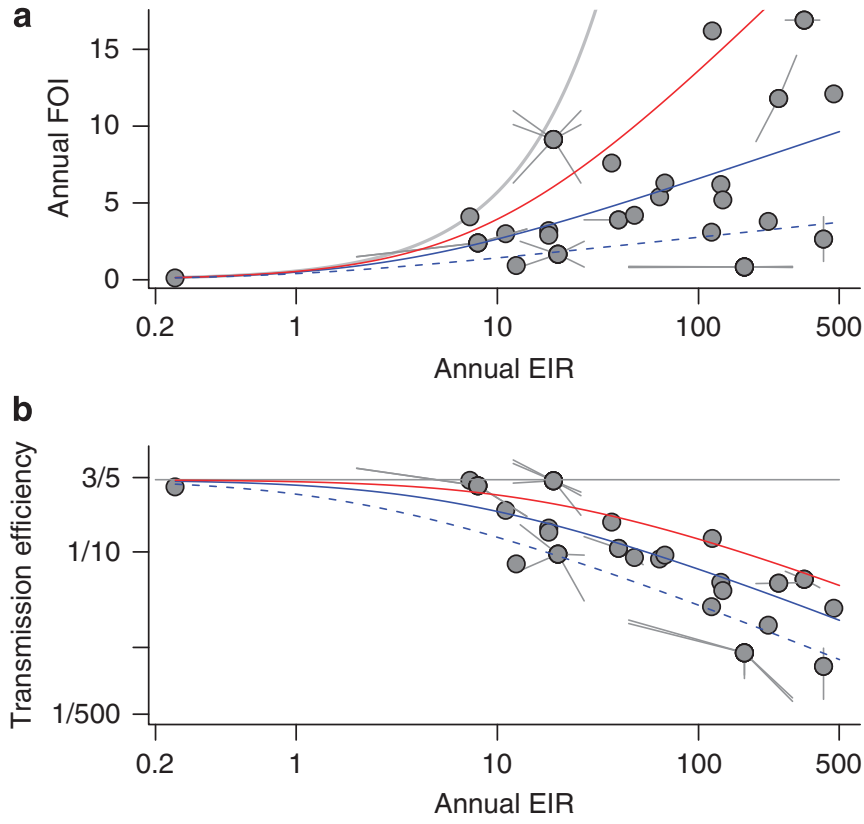

Figure 3 | The annual FOI and transmission efficiency estimated from cross-sectional PR surveys. The data have been plotted in two ways: (a) the estimated annual FOI and (b) transmission efficiency plotted versus the log of EIR. Each point represents a single study. When several estimates of the EIR or the FOI were reported, a 'spider' was plotted with its centre at the arithmetic mean and legs that connect the center to each one of the estimates. The solid blue line shows a good fit to the data using Equation 1, plotted with $b=0.55, \alpha=4.6$ and $t=43$ days. The dashed blue line was drawn with longer times and much higher degrees of heterogeneous biting (that is, with $\alpha=10$ and $t=60$ days). Also plotted in red is the heterogeneous biting model fitted to the synthetic cohort study, which serves as an upper bound for this data. The grey line is plotted for reference to show the relationship predicted by the Ross-Macdonald model, assuming $b=0.55$.

rate (PR), highly stratified by age in children $<2$ years ${ }^{12,14,19}$. A search found 23 suitable studies with paired estimates of the EIR (Supplementary Note 2). The age-stratified PR data were then analysed by fitting simple 'catalytic' models using maximum likelihood (Methods).

The estimates of FOI and transmission efficiency plotted with respect to the EIR revealed a similar pattern to that found in the synthetic cohort study (Fig. 3). The FOI saturates when the annual EIR exceeds $\sim 10$, so there is also a sharp decline in transmission efficiency. This pattern is also a robust property of the heterogeneous biting model. To demonstrate a correspondence between the shape of the heterogeneous biting model and the observed pattern, a model fitting exercise was performed with the heterogeneous biting model (Fig. 3, Methods). The model of heterogeneous biting describes the scatter between the EIR and the crude estimates of the FOI with the MLE parameters from the Saradidi study and $t=43$ days.

Cross-sectional serological surveys. Serology and parasitology were measured in cross-sectional surveys of 21 populations, and for the same populations the EIR was either measured or inferred as a function of elevation ${ }^{34}$. These studies thus provide a unique opportunity to examine serology and to compare it directly with parasitology. The SCR was estimated in children between the age of 1 and 10 years by looking at the proportion of individuals who were positive for at least one of the three serological markers: AMA, MSP1 or MSP2 (Fig. 4, Methods) ${ }^{35,38}$.

In principle, the SCR is analogous to the FOI, but the two measures would only be expected to correspond if every infection 
a
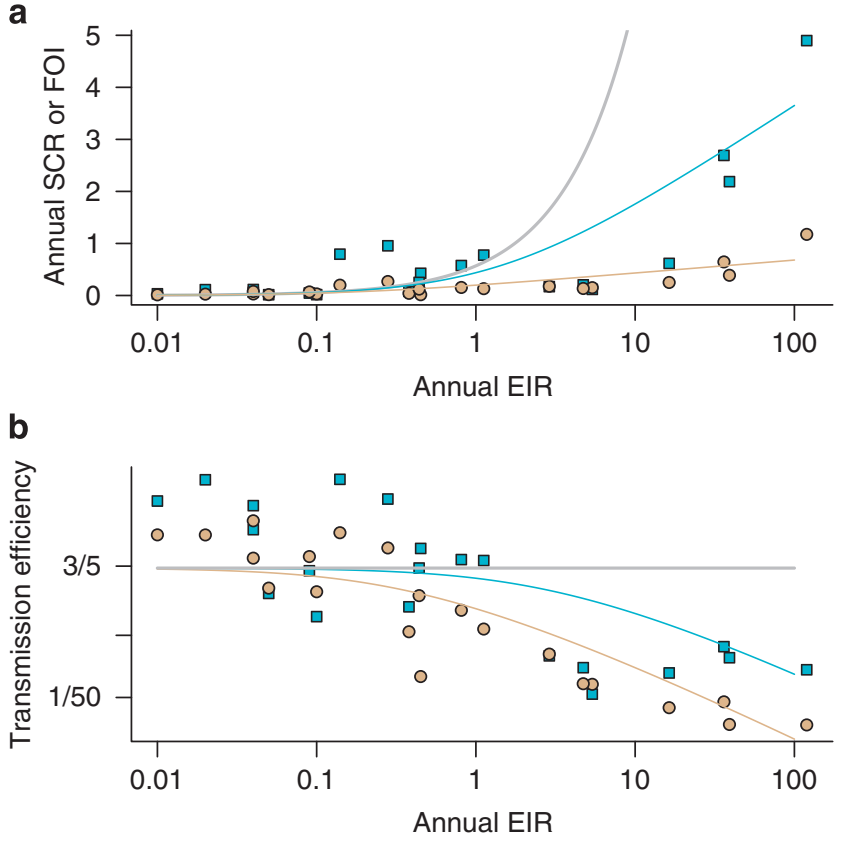

Figure 4 | Analysis of a set of linked cross-sectional serological and parasite surveys. The data have been plotted in two ways:

(a) the estimated annual SCR (tan circles) and FOI (cyan squares) and (b) transmission efficiency based on these estimates plotted versus the log of EIR. The tan line (SCR) was plotted with $b=0.55, \alpha=4.6$ and $t=2$ years, and the cyan line (FOI by PfPR) was plotted with $b=0.55$, $\alpha=4.6$ and $t=0.25$ years. Also plotted is the predicted relationship from the Ross-Macdonald model, assuming $b=0.55$ (grey)

generated an immunological response to the antigen(s) being tested. If immunity is heterogeneous, that is, if some individuals do not respond to the focal antigen, then the SCR will be lower than the FOI when measured by a single antigen, but for homogeneous serological responses or for a sufficiently large panel of heterogeneous serological responses, the SCR and the FOI should correspond. Notably, the estimates of SCR using a response to at least one of three markers were higher than the published estimates of the SCR using only MSP1 (ref. 34).

In these studies, seroprevalence in children was higher than prevalence, but estimates of the FOI were, on average, five times higher than the estimates of SCR. Serology in principle provides a robust marker of previous infection, after considering heterogeneous immunity, because the decay of an immunological response is slow $^{34}$, especially compared with infections that clear relatively quickly. With heterogeneous immunity, higher seroprevalence can produce lower estimates of SCR compared with the estimates of the FOI because parasite clearance rates $(r \approx 1.8$ per year $)$ are $\sim 100$ times higher than the estimated decay of seropositivity $(\lambda \approx 0.017$ per year for MSP 1$)^{34}$. The model of heterogeneous biting was fit to the data by finding a time interval, as performed above. Good fits were found with $t=2$ years (SCR) and 0.25 years (FOI).

Other longitudinal studies. In addition to the synthetic cohort studies, other longitudinal studies have estimated attack rates by following individuals over time as they naturally acquired and cleared infections. One longitudinal study in Idete, Tanzania, followed children $<1$ year of age for 2 weeks ${ }^{36}$; attack rates were computed from individuals who were initially negative for parasites. Another longitudinal study conducted in Garki, Nigeria, followed individuals over time as they naturally acquired and cleared infections, and these data were used to estimate the FOI and the

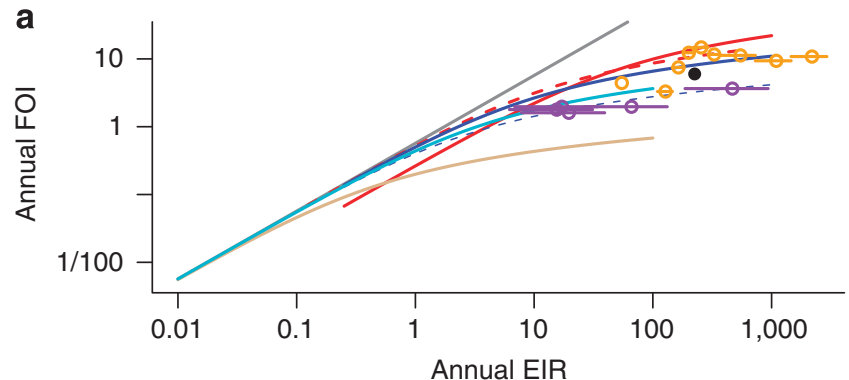

b

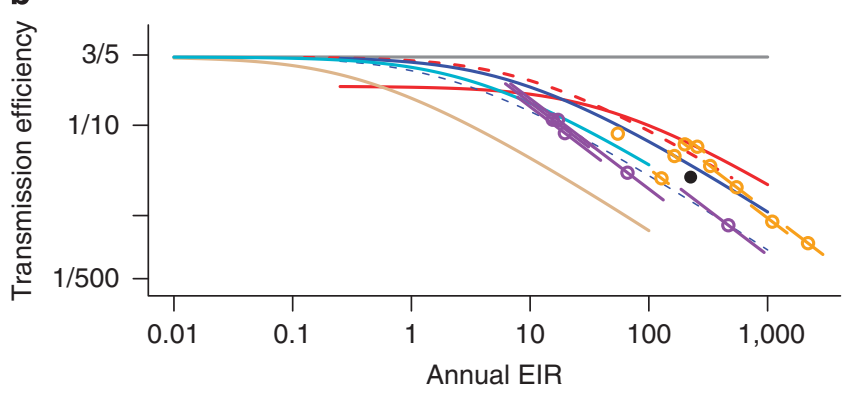

Figure 5 | Estimated annual FOI and transmission efficiency from all the studies plotted versus annual EIR. The data have been plotted in two ways: (a) FOI data from three longitudinal studies in Idete (orange), Garki (purple) and Dielmo (black) have been plotted without further analysis versus the annual EIR on log-log scale and (b) transmission efficiency was also plotted for those same studies. Also plotted are the Ross-Macdonald model assuming $b=0.55$ (grey), the reanalysis of the synthetic cohort study (red, see Figure 2), the fit to the estimated FOI from the crosssectional PR surveys (blue, see Figure 3 ) and the fits to estimated FOI and SCR from the paired cross-sectional surveys (cyan and tan, see Figure 4).

clearance rate ${ }^{15}$. A third study visited 94 children in Dielmo village, Senegal, every day over 4 months; all malaria attacks were observed and treated ${ }^{37}$.

Depending on how a longitudinal study has been carried out, the estimates are prone to detection errors because parasite densities vary over the course of an infection affecting the sensitivity and specificity of microscopy ${ }^{15,36}$. Medical interventions can also interfere with the estimates if, for example, residual drug levels delay new attacks ${ }^{37}$. Despite the caveats, the data have been plotted without further analysis for comparison; they are broadly consistent with each other and with the other studies (Fig. 5).

Cross-study comparisons. The data from the longitudinal studies have been plotted along with the Ross-Macdonald model, the trend lines from the synthetic cohort study, the cross-sectional parasite surveys and the cross-sectional serological surveys (Fig. 5). Across the spectrum of transmission intensity, the assembled data show that transmission efficiency varied enormously, associated strongly, negatively and nonlinearly with the EIR and differed systematically depending on the method used to measure it.

Under the ideal conditions of experimental challenge in previously unexposed humans, $\sim 55 \%$ of infectious bites caused an infection (roughly half). In the synthetic cohort study, $\sim 5 \%$ of infectious bites produced an infection (that is, 1 in 20), but efficiency varied from $\sim 20 \%$ down to $1.4 \%$ (that is, from 1 in 5 to 1 in 70 ), and there was a nonlinear association with the $\mathrm{EIR}^{16}$. Highly variable estimates were found when the FOI was estimated using cross-sectional parasite surveys. An extreme estimate was $\sim 0.3 \%$ of infectious bites producing an infection (that is, 1 in 350). Very similar patterns were found in longitudinal studies. Finally, for the same EIR, transmission efficiency was lower in the serological studies by a factor of five. 
All these patterns arise because the annual FOI saturates at high values of the EIR, so that there were at most 15 infections per person per year.

\section{Discussion}

The difference between the EIR and the FOI has been measured, rediscovered and discussed several times ${ }^{12,14,17,19,20}$. Here, estimates of the EIR and the FOI have been assembled to show that there is a robust pattern between transmission efficiency and intensity, that the quantitative effect is large and that it is important for understanding and modelling transmission. Patterns in the assembled data raise three questions: what explains the strong association between transmission intensity and transmission efficiency; what explains the systematic differences in the estimates of transmission efficiency obtained through different methods; and finally, what explains the variability among studies? There are three dominant and non-exclusive hypotheses for the association between transmission efficiency and the EIR: immunity, heterogeneous biting and systematic bias in the estimation of the EIR.

Bias in estimating the EIR could explain some of the pattern; several common methods of estimating the EIR are known to be biased relative to one another ${ }^{39,40}$. A bias in EIR could explain why the highest transmission efficiency in Saradidi was approximately half of that observed for experimental challenge to infectious mosquitoes. In that study, adults were used as bait to attract and trap the mosquitoes, but attack rates were measured in children; an observed correlation between body size and biting rates is of the right magnitude to explain the gap ${ }^{41,42}$. A similar bias would also affect the other studies. A bias in the EIR could only explain the nonlinear patterns in transmission efficiency if the magnitude of the bias increased sharply with the EIR. The studies assembled and analysed here cannot test whether such a systematic bias exists.

It is possible, on the other hand, to form stronger conclusions about the relative merits of immunity. In particular, long-lasting immunity does not seem to be the main reason why transmission is inefficient. In the Saradidi study reanalysed here, in cohorts drawn at random from the same population but started at different times, transmission efficiency varied every 2 weeks and it was correlated with the EIR. Long-lasting immunity would not predict any association between transmission efficiency and the time of year when a random cohort was drawn. Other studies have come to similar conclusions about the lack of infection blocking immunity early in childhood ${ }^{17,43}$, although it might still develop in adults ${ }^{17}$. Bloodstage immunity is unlikely to be a factor in the young children considered in these studies, but it could be a factor in older children or adults: one study found that malaria attacks were less common in older children ${ }^{37}$, but another found very similar attack rates in children and adults ${ }^{32,33}$. An acute immune system response, such as to mosquito biting, could explain the variability in transmission efficiency. Curiously, estimates of the EIR 6-8 weeks previously were weakly associated with transmission efficiency, but there was no association with the EIR 2-6 or 8-10 weeks previously. In sum, immunity does not seem to explain inefficient transmission in very young children.

Heterogeneous biting provides a simple, plausible and parsimonious explanation for some part of the nonlinear relationship between the EIR and the FOI. A model of heterogeneous biting, where $20 \%$ of the population gets $80 \%$ of the bites, is consistent with the EIRcorrelated temporal variability in transmission efficiency observed in Saradidi, and with the patterns of transmission efficiency and intensity observed across the range of other studies described here. Heterogeneous biting also parsimoniously explains the empirical relationship between the EIR and the PR in older children ${ }^{27}$.

Enormous variability in transmission efficiency relative to transmission intensity could be attributed to the degree of heterogeneous biting, or to a range of other factors, including the methods used to estimate the EIR or the FOI. Estimates of the EIR differ in predictable ways, depending on the methodology and frequency with which human biting rate and sporozoite rate are measured ${ }^{39,40,44-47}$. An additional explanation for the variability is that the efficiency of transmission differs among vector species ${ }^{48}$. The experimental challenges were almost universally conducted with infectious Anopheles stephensi, but most of the studies were conducted in Africa, where the An. gambiae complex predominates. One study, which was conducted in Madang, Papua New Guinea, had different vectors and extremely low efficiency of transmission. The Madang study was also the only study conducted, where Plasmodium vivax was highly prevalent, and, of all the studies, it had the most inefficient transmission.

The lowest transmission efficiency was found using serological data, a pattern that gave rise to one of the challenges to the theory ${ }^{11}$. The analysis of the SCR by one of three different antigens produced higher estimates than the analysis for any one antigen, consistent with human immune responses that are highly heterogeneous: humans in a population often have a measurable serological response to some antigens but not others (that is, AMA positive, but MSP1 negative). A more relevant measure in this case may be the immune response to whole parasite antigens (either intact or lysed) rather than specific antigens. The more pressing question for this study is whether heterogeneous immunity to malaria is sufficient to explain the range of immune responses and the levels of transmission efficiency that were observed.

Heterogeneous biting has broad implications for malaria epidemiology and control, for the evolutionary ecology of malaria parasites and for other mosquito-borne diseases. Aggregated exposure leads to superinfection, which could also affect other mosquitoborne diseases with multiple circulating serotypes, such as dengue. Associated parasite populations will tend to be highly aggregated among humans, and infections will be highly genetically diverse affecting parasite population genetics and parasite life history traits, including gametocytogenesis, the evolution of resistance to antimalarial drugs and the evolution of virulence.

Heterogeneous biting potentially confounds every measure of malaria in epidemiology and public health, from estimates of the burden of malaria to individual risk factors for clinical or severe malaria. When exposure to malaria within a defined study population is heterogeneous, it may be possible to find individuals in that population from across the entire spectrum of exposure to malaria. If the epidemiological effects of high exposure saturate in individuals, then the functional effects of heterogeneous distributions of exposure can be larger than the effect of the average level of exposure; for example, if the risk of severe malaria and mortality were similar for children receiving either 10,100 or 1,000 infectious bites in a year, then the incidence of severe malaria and malaria mortality in a population would be proportional to the number of children receiving more than 10 infectious bites per year. Populations with the same average levels of exposure can thus have very different outcomes, and populations with very different average levels of exposure can have very similar outcomes. Exposure to malaria has consistently been proposed as a cause of immunoepidemiological patterns and clinical outcomes of malaria across the transmission intensity spectrum ${ }^{22,49,50}$, for example, but similar to the relationship between the EIR and the FOI described here, the empirical relationships are highly variable and saturate at high intensity ${ }^{51}$. Heterogeneous biting could also be an underlying cause of the variability in measures of malaria disease and burden.

The discrepancy between the EIR and the FOI is historically important and relevant for malaria transmission dynamics and control, because metrics of malaria transmission are used to stratify risk, plan for control and evaluate the responses to control. In fact, many of these studies assembled here were performed to establish baseline patterns of transmission to prepare for vaccine trials. Malaria transmission models provide a coherent framework 
for describing the nonlinear relationships among malaria metrics and the reductions in transmission that are required to achieve the objectives of malaria control or elimination. For control, a useful metric is the basic reproductive number, $\mathrm{R}_{0}$, which describes reductions in transmission required to control and ultimately eliminate malaria ${ }^{4,8,9}$. Practical methods for estimating $\mathrm{R}_{0}$ rely on having malaria transmission models that can accurately describe the relationships among the most commonly measured metrics of malaria across the natural spectrum of transmission intensity, such as the $\mathrm{PR}$, the EIR, the FOI or the $\mathrm{SCR}^{4,8,9}$. An often-overlooked aspect of transmission models is the high variability in mosquito population densities over space and time that contribute to highly focal transmission and heterogeneous biting. In this study, we have shown that the assumptions of the Ross-Macdonald model are violated, and the predictions are highly inaccurate when the EIRs exceed more than approximately one infectious bite per person per year. Because measures of the EIR are systematically much higher than the measures of the FOI, estimates of $\mathrm{R}_{0}$ based on the EIR are much higher than the estimates based on the FOI. The enormous discrepancy between isolated estimates of $\mathrm{R}_{0}$ made by the EIR and the FOI raised questions about the quantitative validity and utility of a vector-based theory of transmission ${ }^{10,11}$. Our analysis suggests that the discrepancy between the FOI and the EIR is to be expected and that the two metrics are consistent under a model of heterogeneous biting. The analysis also suggests that heterogeneous biting explains some part of the discrepancy between the SCR and the EIR after additionally considering heterogeneous immunity.

Simple mathematical models and comparative studies can help provide a solid theoretical basis for measuring and interpreting the transmission intensity of mosquito-borne diseases using standard metrics, such as the EIR, the PR and the SCR. The standard interpretation of $\mathrm{R}_{0}$ will need revision, however, with new modelling frameworks that consider the causes and patterns of heterogeneous biting. The data assembled in this study suggest that entomological measures and the associated estimates of $\mathrm{R}_{0}$ still serve as a guideline for setting vector control targets, but that entomological and epidemiological measures deviate when the EIR exceeds approximately one infectious bite per person per year. $\mathrm{R}_{0}$ may not describe the expected number of people who would be infected after one parasite generation or the rate of increase in the number of cases during an epidemic because the infectious bites are inefficiently distributed ${ }^{4}$. Models do, however, provide an indispensable tool for integrating information and leveraging further insight from large databases of parasite prevalence ${ }^{52}$ when combined with methods for reconstructing the history of transmission ${ }^{34}$ to provide an evidence-base and theoretical support for making decisions across the spectrum, from endemicity to the interruption of transmission ${ }^{53}$.

\footnotetext{
Methods

Literature search. A search was developed from a core set of papers and expanded using PubMed. The core papers were a review by Epstein et al..$^{29}$; the study from Saradidi, Kenya, by Beier et al. ${ }^{16}$; historical papers estimating the FOI from ageprevalence relations ${ }^{14,19}$; a recent review of studies that reported both the EIR and the $\mathrm{PR}^{54}$; a published description of EIR versus $\mathrm{SCR}^{34}$; and a longitudinal study conducted during the Garki project ${ }^{15}$. Search criteria were expanded to find other kinds of studies that had estimated malaria incidence by using a combination of terms, including 'malaria attack', 'malaria incidence,' 'entomological' and 'force of infection.' The five categories were finalized, and search methods turned to expanding the list of papers within each category. The R code used to generate all of the figures is freely available upon request.
}

Experimental challenge. Data in Table 1 were assembled starting from recent reviews ${ }^{28-31}$ to find a complete set of individuals challenged to the bites of infectious mosquitoes. The search process and data sources are in the Supplementary Information. When a study reported the exact number of bites, it was considered to be both the upper and the lower bound.

A synthetic cohort study. Data from Beier et al. ${ }^{16}$ were extracted to obtain the number in each cohort during each observation period, and the number that became positive. The proportion of a cohort that was positive is called the attack rate. In a homogeneous population stratum, the FOI is the hazard rate for infection, and it is related to the attack rate, $A$ (the proportion of a cohort that becomes infected after $t$ days) by the formula $A(t)=1-e^{-h t}$. The FOI, $h$, can be computed from an attack rate by taking the inverse relationship:

$$
h=\frac{-\log (1-A(t))}{t} .
$$

When biting is heterogeneous, the daily EIR varies and defines a different relationship between the FOI and attack rates. The heterogeneous biting model used here assumes that biting weights (that is, $\omega$ ) have a Gamma distribution with a mean of one and a variance of $\alpha$. The biting rates, therefore, would have a Gamma distribution with the mean EIR and a squared coefficient of variation $\alpha$, called the index of heterogeneous biting. After $t$ days, the proportion of a cohort that was infected is:

$$
\int_{0}^{\infty} \Gamma(\omega, \alpha)\left(1-\mathrm{e}^{-\omega b E t}\right) \mathrm{d} \omega=1-(1+\alpha b E t)^{-1 / \alpha} .
$$

Observations were omitted from the analysis if the observed EIR was zero; likelihood returned an error when predicted attack rates were exactly zero but observed attack rates were positive.

To account for extra-binomial variation in attack rates, the log likelihood for a set of model-based predicted probabilities, $\left\{x_{i}\right\}$, given the corresponding sets of $\left\{P_{i}\right\}$ positive and $\left\{N_{i}\right\}$ negative observations was computed using the function:

$$
L L=\sum_{i} \log \left(\beta\left(x_{i} \theta+P_{i},\left(1-x_{i}\right) \theta+N_{i}\right)\right)-\log \left(\beta\left(x_{i} \theta,\left(1-x_{i}\right) \theta\right)\right)
$$

where $\beta$ is the two-parameter family of beta-binomial probability distribution functions. The parameter $\theta$ is fitted along with the function that predicts $\left\{x_{i}\right\}$, the predicted attack rates for the data from Saradidi, Kenya. The AIC $\left(-2^{\star} L L+2 k\right.$, where $k$ is the number of parameters) was used to compare the fits of different approximating models ${ }^{55}$.

The MLE was repeated by assuming that the synthetic cohort experienced a real EIR that was a factor $\delta$ lower than the estimated EIR. The putative bias, $\delta$, was varied between 1 and 10 . Increasing $\delta$ initially increases the estimate of $b$, but after $b$ reaches a maximum value, then a further increase in $\delta$ reduces the estimates of $\alpha$, indicating less biting heterogeneity.

The analysis was also repeated with the formula $\mathrm{EIR}=\mathrm{EIR}_{1}+\xi \mathrm{EIR}_{2}$, where the EIR in the previous 2 weeks was called $\mathrm{EIR}_{1}$, and the EIR in a previous 2-week period was called $\mathrm{EIR}_{2}$. The heterogeneous biting model that considered the EIR $6-8$ weeks before had parameter values of $b=0.71, \alpha=6.6, \xi=-0.05, \theta=11.4$, and it ranked higher than the model that did not consider EIR in previous periods, which had a $\triangle \mathrm{AIC}$ value of 0.96 .

Cross-sectional parasite surveys. The papers by Macdonald ${ }^{19}$, Pull and $\mathrm{Grab}^{14}$ and a recent review of papers that reported both the EIR and the PR since 1980 by Hay et al..$^{54}$ were used as a basis to find any papers that reported both the EIR and PRs highly stratified by age. Additional papers were found by searching bibliographies of the existing publications. Papers were also identified through a PubMed search using the terms 'prevalence' or 'parasite rate' and 'entomological inoculation rate' or 'human biting rate.' Papers were included if they reported the PR stratified in at least two age classes in children $<2$ years. New papers were identified periodically after the search and added to the list. For each one of the 23 studies, data were extracted and the likelihood computed at the highest level of stratification reported

Simple catalytic models ${ }^{14}$ were fitted to the data to get a crude estimate of the FOI from the PR $(X)$ as a function of age:

$$
X(a)=\frac{h}{h+r}\left(1-\mathrm{e}^{-(h+r) a}\right) .
$$

It was assumed that $r=0.005$ per day.

In Equation 1, time and the index of heterogeneous biting always appear together, so they are not identifiable. Moreover, cross-sectional surveys do not have a fixed time interval, because exposure accumulates with age. The index of heterogeneous biting was therefore fixed at the fitted value from the Saradidi study (that is, $\alpha \approx 4.6$ ) and the parameter describing time was allowed to vary. The initial bestfit value of $b$ was also fitted initially, but the initial fitted value was non-sensically larger than one. The parameter $b$ was then fixed at the value from the experimental challenge studies (that is, $b=0.55$ ), and the parameter describing the time interval was fit to the scatter plot of the EIR versus transmission efficiency (Fig. 3).

Cross-sectional serological and parasitological surveys. The original data from the study described by Corran et al. ${ }^{34}$ were reanalysed to estimate both the SCR and the FOI. Analysis of SCR also uses Equation 5, but different parameter names are typically used for the SCR $(\lambda)$ and the FOI $(h)$, and for the decay rate of seropositivity $(\rho)$ rather than parasite clearance rates $(r)$. Malaria parasites are not 
transmitted vertically, from mother to child, but maternal antibodies are often present in infants, so our estimates of the SCR ignored infants.

Longitudinal studies. A search for other longitudinal studies was undertaken using the terms 'malaria incidence' and 'entomological inoculation rate' and 'longitudinal' or 'force of infection' and 'entomological inoculation rate' and 'longitudinal.' No additional papers were found in this way. Later, two other papers were discovered in other ways and added to the analysis ${ }^{36,37}$. In the Garki study, estimates of the human biting rate were reported, the number of bites by potential vectors per person per day. Estimates of the EIR were made by assuming that the sporozoite rate, the fraction of infectious mosquitoes, was $2.5 \%$, with a range between 1 and $5 \%$. The plotted data from Dielmo included only children $<7$ years.

\section{References}

1. Ross, R. The Prevention of Malaria 2nd edn (John Murray, 1911)

2. Macdonald, G. The Epidemiology and Control of Malaria (Oxford University Press, 1957)

3. Lotka, A. J. Contributions to the analysis of malaria epidemiology. Am. J. Hyg 3, 1-121 (1923).

4. Smith, D. L., McKenzie, F. E., Snow, R. W. \& Hay, S. I. Revisiting the basic reproductive number for malaria and its implications for malaria control. PLoS Biol. 5, e42 (2007).

5. Garrett-Jones, C. Prognosis for interruption of malaria transmission through assessment of the mosquito's vectorial capacity. Nature 204, 1173-1175 (1964)

6. Macdonald, G. The analysis of malaria epidemics. Trop. Dis. Bull. 50, 871-889 (1953).

7. Macdonald, G. Epidemiological basis of malaria control. Bull. World Health Organ. 15, 613-626 (1956).

8. Smith, D. L., Hay, S. I., Noor, A. M. \& Snow, R. W. Predicting changing malaria risk after expanded insecticide-treated net coverage in Africa. Trends Parasitol. 25, 511-516 (2009).

9. Dietz, K. The estimation of the basic reproduction number for infectious diseases. Stat. Methods Med. Res. 2, 23-41 (1993).

10. Najera, J. A. A critical review of the field application of a mathematical model of malaria eradication. Bull. World Health Organ. 50, 449-457 (1974).

11. Gupta, S., Trenholme, K., Anderson, R. M. \& Day, K. P. Antigenic diversity and the transmission dynamics of Plasmodium falciparum. Science 263, 961-963 (1994).

12. Davey, T. H. \& Gordon, R. M. The estimation of the density of infective anophelines as a method of calculating the relative risk of inoculation with malaria from different species or in different localities. Ann. Trop. Med. Parasit. 27, 27-52 (1933).

13. Davidson, G. \& Draper, C. C. Field studies of some of the basic factors concerned in the transmission of malaria. Trans. R Soc. Trop. Med. Hyg. 47, 522-535 (1953).

14. Pull, J. H. \& Grab, B. A simple epidemiological model for evaluating the malaria inoculation rate and the risk of infection in infants. Bull. World Health Organ. 51, 507-516 (1974)

15. Bekessy, A., Molineaux, L. \& Storey, J. Estimation of incidence and recovery rates of Plasmodium falciparum parasitaemia from longitudinal data. Bull. World Health Organ. 54, 685-693 (1976).

16. Beier, J. C. et al. Plasmodium falciparum incidence relative to entomologic inoculation rates at a site proposed for testing malaria vaccines in western Kenya. Am. J. Trop. Med. Hyg. 50, 529-536 (1994).

17. Smith, T. et al. Relationship between the entomologic inoculation rate and the force of infection for Plasmodium falciparum malaria. Am. J. Trop. Med. Hyg. 75, 11-18 (2006)

18. Smith, T. A. Estimation of heterogeneity in malaria transmission by stochastic modelling of apparent deviations from mass action kinetics. Malar. J. 7, 12 (2008).

19. Macdonald, G. The analysis of malaria parasite rates in infants. Trop. Dis. Bull. 47, 915-938 (1950).

20. Pringle, G. Some factors affecting the detection of residual transmission in malaria eradication schemes in Africa. Bull. World Health Organ. 30, 858-862 (1964).

21. Pringle, G., Draper, C. C. \& Clyde, D. F. A new approach to the measurement of residual transmission in a malaria control scheme in East Africa. Trans. R Soc. Trop. Med. Hyg. 54, 434-438 (1960).

22. Smith, T., Killeen, G., Lengeler, C. \& Tanner, M. Relationships between the outcome of Plasmodium falciparum infection and the intensity of transmission in Africa. Am. J. Trop. Med. Hyg. 71, 80-86 (2004)

23. Doolan, D. L. \& Martinez-Alier, N. Immune response to pre-erythrocytic stages of malaria parasites. Curr. Mol. Med. 6, 169-185 (2006).

24. John, C. C. et al. Correlation of high levels of antibodies to multiple preerythrocytic Plasmodium falciparum antigens and protection from infection. Am. J. Trop. Med. Hyg. 73, 222-228 (2005).

25. Donovan, M. J. et al. Uninfected mosquito bites confer protection against infection with malaria parasites. Infect. Immun. 75, 2523-2530 (2007).
26. Woolhouse, M. E. et al. Heterogeneities in the transmission of infectious agents: implications for the design of control programs. Proc. Natl Acad. Sci. USA 94, 338-342 (1997).

27. Smith, D. L., Dushoff, J., Snow, R. W. \& Hay, S. I. The entomological inoculation rate and Plasmodium falciparum infection in African children. Nature $\mathbf{4 3 8}$ 492-495 (2005)

28. Hoffman, S. L. Experimental challenge of volunteers with malaria. Ann. Intern. Med. 127, 233-235 (1997).

29. Epstein, J. E. et al. Safety and clinical outcome of experimental challenge of human volunteers with Plasmodium falciparum-infected mosquitoes: an update. J. Infect. Dis. 196, 145-154 (2007).

30. Rickman, L. S. et al. Plasmodium falciparum-infected Anopheles stephensi inconsistently transmit malaria to humans. Am. J. Trop. Med. Hyg. 43, 441-445 (1990).

31. Church, L. W. et al. Clinical manifestations of Plasmodium falciparum malaria experimentally induced by mosquito challenge. J. Infect. Dis. 175, 915-920 (1997).

32. Owusu-Agyei, S. et al. Incidence of symptomatic and asymptomatic Plasmodium falciparum infection following curative therapy in adult residents of northern Ghana. Am. J. Trop. Med. Hyg. 65, 197-203 (2001).

33. Baird, J. K. et al. Seasonal malaria attack rates in infants and young children in northern Ghana. Am. J. Trop. Med. Hyg. 66, 280-286 (2002).

34. Corran, P., Coleman, P., Riley, E. \& Drakeley, C. Serology: a robust indicator of malaria transmission intensity? Trends Parasitol. 23, 575-582 (2007).

35. Drakeley, C. J. et al. Estimating medium- and long-term trends in malaria transmission by using serological markers of malaria exposure. Proc. Natl Acad. Sci. USA 102, 5108-5113 (2005).

36. Charlwood, J. D. et al. Incidence of Plasmodium falciparum infection in infants in relation to exposure to sporozoite-infected anophelines. Am. J. Trop. Med. Hyg. 59, 243-251 (1998).

37. Rogier, C. \& Trape, J. F. Malaria attacks in children exposed to high transmission: who is protected? Trans. $R$ Soc. Trop. Med. Hyg. 87, 245-246 (1993).

38. Grab, B. \& Pull, J. H. Statistical considerations in serological surveys of population with particular reference to malaria. J. Trop. Med. Hyg. 77, 222-232 (1974).

39. Mathenge, E. M. et al. Comparative performance of the Mbita trap, CDC light trap and the human landing catch in the sampling of Anopheles arabiensis, An. funestus and culicine species in a rice irrigation in western Kenya. Malar. J. 4, 7 (2005).

40. Mathenge, E. M. et al. Comparative field evaluation of the Mbita trap, the Centers for Disease Control light trap, and the human landing catch for sampling of malaria vectors in western Kenya. Am. J. Trop. Med. Hyg. 70, 33-37 (2004)

41. Carnevale, P., Frezil, J. L., Bosseno, M. F., Le Pont, F. \& Lancien, J. The aggressiveness of Anopheles gambiae $A$ in relation to the age and sex of the human subjects. Bull. World Health Organ. 56, 147-154 (1978).

42. Port, G. R., Boreham, P. F. L. \& Bryan, J. H. The relationship of host size to feeding by mosquitoes of the Anopheles gambiae giles complex (Diptera: Culicidae). Bull. Entomological Res. 70, 133-144 (1980).

43. Kitua, A. Y. et al. Antibodies against Plasmodium falciparum vaccine candidate in infants in an area of intense and perennial transmission: relationships with clinical malaria and with entomological inoculation rates. Parasite Immunol. 21, 307-317 (1999).

44. Kelly-Hope, L. A. \& McKenzie, F. E. The multiplicity of malaria transmission: a review of entomological inoculation rate measurements and methods across sub-Saharan Africa. Malar J. 8, 19 (2009).

45. Hay, S. I., Rogers, D. J., Toomer, J. F. \& Snow, R. W. Annual Plasmodium falciparum entomological inoculation rates (EIR) across Africa: literature survey, internet access and review. Trans. R Soc. Trop. Med. Hyg. 94, 113-127 (2000).

46. Filion, G. J. P., Paul, R. E. L. \& Robert, V. Transmission and immunity: the importance of heterogeneity in the fight against malaria. Trends Parasitol. 22 345-348 (2006).

47. Drakeley, C. et al. An estimation of the entomological inoculation rate for Ifakara: a semi-urban area in a region of intense malaria transmission in Tanzania. Trop. Med. Int. Health 8, 767-774 (2003)

48. Kiszewski, A. E. \& Teklehaimanot, A. A review of the clinical and epidemiologic burdens of epidemic malaria. Am. J. Trop. Med. Hyg. 71, 128-135 (2004)

49. Snow, R. W. et al. Relation between severe malaria morbidity in children and level of Plasmodium falciparum transmission in Africa. Lancet 349, 1650-1654 (1997).

50. Snow, R. W. \& Marsh, K. The consequences of reducing transmission of Plasmodium falciparum in Africa. Adv. Parasitol. 52, 235-264 (2002)

51. Rowe, A. K. et al. The burden of malaria mortality among African children in the year 2000. Int. J. Epidemiol. 35, 691-704 (2006).

52. Hay, S. I. et al. A world malaria map: Plasmodium falciparum endemicity in 2007. PLoS Med. 6, e1000048 (2009). 
53. Hay, S. I., Smith, D. L. \& Snow, R. W. Measuring malaria endemicity from intense to interrupted transmission. Lancet Infect. Dis. 8, 369-378 (2008).

54. Hay, S. I., Guerra, C. A., Tatem, A. J., Atkinson, P. M. \& Snow, R. W. Urbanization, malaria transmission and disease burden in Africa. Nat. Rev. Microbiol. 3, 81-90 (2005).

55. Burnham, K. P. \& Anderson, D. R. Model Selection and Multi-Model Inference, A Practical Information-Theoretic Approach 2nd edn (Springer-Verlag, 2002).

\section{Acknowledgments}

We thank Arnaud Le Menac'h, Kevin Marsh, Carlos Guerra, Philip Bejon, Andy Tatem, Anand Patil, Pete Gething and Heather Randell for help in extracting data. Thanks to Rob Hermsen, from The Radboud University Nijmegen Medical Centre, The Netherlands, for providing information about the exact number of infectious bites and the overlap between individuals reported in studies conducted there. D.L.S. is supported by a grant from the Bill \& Melinda Gates Foundation (\#49446). C.J.D. is supported by the Wellcome Trust (\#078925). S.I.H. is supported by a Senior Research Fellowship from the Wellcome Trust (\#079091). C.C. was supported by the UF Science for Life Program, funded by the Howard Hughes Medical Institute. D.L.S. and S.I.H. were also supported by the RAPIDD program of the Science \& Technology Directorate, Department of Homeland Security, and the Fogarty International Center, National Institutes of Health. D.L.S. and C.C. acknowledge the support of the Emerging Pathogens Institute, University of Florida. This work also forms part of the output of the Malaria Atlas Project (MAP, http://www.map.ox.ac.uk), principally funded by the Wellcome Trust, UK.
The funders had no role in study design, data collection and analysis, decision to publish or preparation of the manuscript.

\section{Author contributions}

D.L.S. designed the study. D.L.S. and S.I.H. wrote the first draft of the manuscript. D.L.S., C.C. and C.J.D. collated and analysed the data. D.L.S. made the graphs. All authors contributed substantively to the writing, revising and editing of the final draft.

\section{Additional information}

Supplementary Information accompanies this paper on http://www.nature.com/ naturecommunications

Competing financial interests: The authors declare no competing financial interests.

Reprints and permission information is available online at http://npg.nature.com/ reprintsandpermissions/

How to cite this article: Smith, D. L. et al. A quantitative analysis of transmission efficiency versus intensity for malaria. Nat. Commun. 1:108 doi: 10.1038/ncomms1107 (2010)

License: This work is licensed under a Creative Commons Attribution-NonCommercialShare Alike 3.0 Unported License. To view a copy of this license, visit http:// creativecommons.org/licenses/by-nc-sa/3.0/ 